\title{
Elevación de seno VS Implante cigomático. Revisión bibliográfica y caso clínico.
}

\author{
Sinus Elevation VS Zygomatic Implant. Case report and review.
}

\author{
Baoluo Gao, L., Baca González, A., Helm González, JG.
}

\section{RESUMEN}

Los tratamientos implantológicos se consideran actualmente una forma predecible para la rehabilitación de los pacientes desdentados, pero muchas veces, debido a la gran reabsorción que sufre el maxilar, requieren de técnicas quirúrgicas avanzadas para ello. El presente trabajo está motivado por la ausencia de un protocolo clínico claro sobre cuándo se debe emplear la elevación sinusal o los implantes cigomáticos en estos pacientes. Para ello, se realiza una búsqueda bibliográfica de los últimos 20 años. Los resultados señalan que la mayor controversia se encuentra en los pacientes que presentan menos de $4 \mathrm{~mm}$ de altura ósea. En el caso descrito, después de estudiar la anchura, altura ósea y el tamaño del seno remanente, además de sus preferencias personales, se realizó el tratamiento implantológico mediante implantes cigomáticos. De la preparación y experiencia de cada cirujano dependerá también el ofrecer una u otra técnica.

Palabras clave: Elevación de seno, implante cigomático, maxilar atrófico, Implante dental.

\begin{abstract}
Implant treatment has become a predictable rehabilitation of the edentulous patient. But on many occasions, due to a great amount of resorption of the maxilla, a clinician has no choice but to rely on advanced surgical techniques. The present study is based on the absence of a clear
\end{abstract}


clinical protocol on when a sinus floor elevation or zygomatic implants should be used. Therefore, a review of the literature up to the past twenty years was conducted. Results show that controversy arises in cases of patients with less than $4 \mathrm{~mm}$ of vertical bone height. On our patient, after evaluating bone width, height and remaining sinus size, as well as the patient's preferences, implant treatment using zygomatic implants was undertaken. However, it should be noted that the choice of one technique or the other shall also depend on the preparation and experience of the surgeon.

Key words: Sinus Elevation, Zygomatic Implant, Maxillary Atrophy, Dental Implant.

\section{INTRODUCCIÓN}

Actualmente en el mundo desarrollado existe un aumento continuo de la esperanza de vida. Hoy en día, España cuenta con un 18,1\% de población por encima de los 65 años y las proyecciones para el futuro son del 33\% en el 2040. INE (2015). La población total o parcialmente desdentada, debido a la edad, siguen en aumento. El maxilar posterior atrófico es una de las zonas de mayor controversia entre los clínicos, por ser su volumen y densidad ósea menores. Esto produce una menor tasa de éxito en implantes. Jaffin et al (1991). Hueso clase E, según Lekholm y Zarb y hueso tipo IV, V y VI según Cawood y Howell son los que requerirán tratamientos más complejos para su rehabilitación implantológica mediante elevación de seno o implantes cigomáticos.

En la actualidad existen dos protocolos quirúrgicos para la rehabilitación implantológica del maxilar atrófico. En caso de un maxilar de más de 10mm de altura, este se rehabilitará con implantes osteointegrados estándares. Cuando existe más de $6 \mathrm{~mm}$, se opta por el uso de implantes cortos o elevación de seno atraumática. Con una altura ósea de 4-6 mm, se empleará elevación de seno con implantes inmediatos. Mitch y Judy (1987). En casos donde exista menos de $4 \mathrm{~mm}$ de altura ósea remanente, será necesario recurrir a elevación de seno más implantes diferidos o con implantes cigomáticos. Fernández et al (2004)

\section{Elevación de seno}

La elevación de la membrana de Schneider para aumentar el espesor del seno maxilar fue descrita por primera vez por Boyne y James. Con un instrumento redondeado, se hacía una antrostomía de unos $10 \mathrm{~mm}$ de diámetro en la pared lateral del seno maxilar. Posteriormente, la membrana se elevaba del suelo del seno, y el espacio que se generaba se rellenaba con un injerto 
de hueso autólogo procedente de la cresta ilíaca del paciente. Tras aproximadamente 3 meses, se colocaban los implantes. Este tipo de técnica se recomienda en casos de reabsorción ósea severa, en la que el espesor remanente es de $4 \mathrm{~mm}$ o incluso menos. Es difícil conseguir una estabilidad primaria en este tipo de pacientes, por lo que no se puede colocar los implantes justo después de hacer la elevación de seno. Más adelante, surgieron los xenoinjerto, sustituyendo en muchos casos el hueso autólogo. Para finalizar, se recubre con una membrana reabsorbible para impedir la proliferación de los fibroblastos en la zona. Este procedimiento conduce a una regeneración ósea en un periodo de 6-8 meses.

\section{Implantes cigomático}

El implante cigomático fue desarrollado por Brånemark en 1989, inicialmente concebido para la rehabilitación de pacientes oncológicos a los que se les practicaba una maxilectomía. Aparicio et al (2012). La principal indicación de este tipo de implante en la actualidad es la rehabilitación del maxilar atrófico. El implante se insertaba desde el aspecto palatino del proceso alveolar, siguiendo la cresta cigomático-alveolar hasta anclarse en el cuerpo del malar, que constituye un excelente pilar por su gran densidad ósea.

El objetivo del presente trabajo es revisar las indicaciones de la elevación sinusal y los implantes cigomáticos para determinar una guía clínica que permita seleccionar la técnica más adecuada para cada paciente aprovechando la presentación de un caso clínico.

\section{MATERIAL Y MÉTODOS}

Se seleccionó artículos científicos de los últimos 20 años y procedentes de los motores de búsqueda MEDLINE ${ }^{\circledR}$, PUBMED ${ }^{\circledR}, \operatorname{SciELO} \AA$, BUCea ${ }^{\circledR}$ y Biblioteca Cochrane ${ }^{\circledR}$. Usando las palabras clave: “Sinus Elevation”, “Zygomatic Implant”, “Maxillary Atrophy”.

Se utilizaron fotografías clínicas previo consentimiento de la paciente.

\section{CASO CLÍNICO}

Paciente mujer de 60 años sin antecedentes médicos de interés con un perfil óseo (Clase E y tipo V). A nivel radiológico, en el primer cuadrante se observa unos valores óseos de $2 \mathrm{~mm}$ de altura y $4 \mathrm{~mm}$ de anchura. En el segundo cuadrante, valores óseos de 3mm de altura y 4mm de anchura. Con estos valores la colocación de implantes osteointegrados convencionales es imposible, por lo que se plantea la realización de elevación de seno más implantes convencionales o implantes cigomáticos. El tratamiento realizado fue la explantación de los 
implantes remanentes y el empleo de tres implantes cigomáticos y cinco convencionales. La cirugía se llevó a cabo bajo sedación consciente en un gabinete quirúrgico. No se registró ninguna complicación quirúrgica ni prostodóncica.

\section{RESULTADOS y DISCUSIÓN}

La tasa de supervivencia a los 10 años entre implante convencional posterior a elevación de seno, e implante cigomático es parecido, con valores de 95,5\% y 96,7\% respectivamente. Schwartz et al (2004). Chrcanovic et al (2013). El uso de los implantes cigomáticos y su éxito a largo plazo ha sido ampliamente documentado y descrito; así, muchos autores consideran que se trata de una técnica quirúrgica adecuada y una alternativa viable en el tratamiento de atrofias óseas en la zona posteriores del maxilar. Davó (2009).

Cabe destacar que la complicación intraoperatoria más común de la elevación de seno es la perforación de la membrana de Schneider (11\%) aunque su perforación no condiciona la supervivencia del implante. Zijderveld. (2008). Por otro lado, la complicación más común en los implantes cigomáticos es la sinusitis recurrente, que se produce en el 2,9\% de las intervenciones. Aparicio et al (2010).

Hay que tener en cuenta también que a las complicaciones intrínsecas de la elevación de seno hay que sumarles las posibles complicaciones que se puedan producir en la fase implantológica, por lo que la literatura señala una menor cantidad de complicaciones en el caso de implantes cigomáticos. Aparicio et al. (2008).

\section{Ventajas de la elevación sinusal con implante diferido frente a implantes cigomáticos:}

- $\quad$ No requiere anestesia general ni sedación.

- Complicaciones más leves comparado con implantes cigomáticos. Schwartz et al. (2004).

- $\quad$ En caso de fracaso, los implantes cigomáticos quedan como opción de rescate.

Ventajas de los implantes cigomáticos frente la elevación de seno con implante diferido:

- $\quad$ Mayor porcentaje de supervivencia. Aparicio et al. (2010).

- $\quad$ Tiempo de tratamiento más reducido.

- $\quad$ Se trata de una técnica menos complicada y con menor riesgo de morbilidad comparado con técnicas que emplean autoinjertos. Chrcanovic et al. (2013).

- $\quad$ Permite llevar a cabo una carga inmediata con una excelente tasa de éxito. Ortiz et al. (2009). 
- $\quad$ Se puede rehabilitar la arcada superior únicamente con 4 implantes cigomáticos. Bedrossian et al. (2009).

\section{Indicaciones de la elevación de seno con implante diferido:}

- Pacientes que por motivos médicos no puedan someterse a una anestesia general o sedación consciente. Boyne y James. (1997).

- $\quad$ Pacientes que no quieran asumir los riesgos de los implantes cigomáticos.

\section{Indicaciones de los implantes cigomáticos:}

- $\quad$ Reabsorción masiva del seno maxilar, tanto a nivel anteroposterior como craneocaudal.

- $\quad$ Cuando no se pueda conseguir estabilidad protésica con cuatro implantes normales pero sí con cuatro implantes cigomáticos. Bedrossian et al. (2009).

- $\quad$ Cuando el paciente requiere un tiempo de tratamiento más rápido.

- $\quad$ Cuando el paciente prefiere someterse únicamente a una cirugía. Fernandez et al. (2004)

\section{CONCLUSIONES}

Ambas técnicas descritas en este trabajo tienen una evidencia científica sólida, avalados por estudios longitudinales con un largo tiempo de seguimiento y unos porcentajes de éxito muy elevados. La controversia en la elección entre ambos tratamientos surge en pacientes con menos de $4 \mathrm{~mm}$ de altura de reborde alveolar. Condicionantes como una anchura y altura ósea mínimas y un seno muy reabsorbido son las principales indicaciones para el empleo de implantes cigomáticos. De la preparación y experiencia de cada cirujano dependerá el ofrecer una u otra técnica.

\section{BIBLIOGRAFÍA}

Aparicio et al. 2006. A Prospective Clinical Study on Titanium Implants in the Zygomatic Arch for Prosthetic Rehabilitation of the Atrophic Edentulous Maxilla with a Follow-Up 5 Years. Clinical Implant Dentistry. 02/2006; 8(3):114-22.

Aparicio et al. 2008. The use of zygomatic implant for prosthetic rehabilitation of the severely resorbed maxilla. Periodontology 2000, Vol. 47, 2008, 162-171

Aparicio et al. 2010. Immediate/Early Loading of Zygomatic Implants: Clinical Experiences after 2 to 5 Years of Follow-up. DOI 10.1111/j.1708-8208.2008.00134

Aparicio et al. 2012 The Long-Term Use of Zygomatic Implants: A 10-Year Clinical and Radiographic Report. Clinical Implant Dentistry and Related Research 
Boyne y James 1997. Sinus augmentation for dental implants: The use of autogenous bone . J Oral Maxillofac Surg. November 1997, Pages 1281-1286.

Branemark et al. 2004. Zygoma fixture in the management of advanced atrophy of the maxilla. Scand $\mathrm{J}$ Plast Reconstr Surg Hand Surg 2004; 38: 70-85

Chrcanovic et al. 2013. Survival and complications of zygomatic implants: A systematic review. Oral Maxillofac Surg (2013) 17:81-93.

Davó. 2009. Zygomatic implants placed with a 2-stage procedure: a 5-year retrospective study. Eur J Oral Implantol 2009; 2(2).

Fernández et al.2004. Implantes transcigomáticos. Avances en Periodoncia. v16. N3, 105-110.

Jaffin et al. 1991. The Excessive Loss of Branemark Fixtures in Type IV Bone: A 5-Year Analysis. Journal of periodontology. 1991. Vol. 62, No. 1, 2-4.

Misch y Judy 1987. Classification of partially edentulous arches for implant dentistry. Int J Oral Implantol. 1987;4(2):7-13.

Schwartz et al. 2004. The prevalence of surgical complications of the sinus graft procedure and their impact on implant survival. J Periodontol • April 2004. 511-516

Zijderveld et al. 2008. Anatomical and Surgical Findings and Complications in 100 Consecutive Maxillary Sinus Floor Elevation Procedures. J Oral Maxillofac Surg 66:14261438, 2008. 